\title{
CONTROLLABILITY OF NONLINEAR NEUTRAL VOLTERRA INTEGRODIFFERENTIAL SYSTEMS
}

\author{
K. BALACHANDRAN and P. BALASUBRAMANIAM ${ }^{1}$
}

(Received 2 July 1992; revised 24 February 1993)

\begin{abstract}
Sufficient conditions for the controllability of nonlinear neutral Volterra integrodifferential systems with implicit derivative are established. The results are a generalisation of the previous results, through the notions of condensing map and measure of noncompactness of a set.
\end{abstract}

\section{Introduction}

Compartmental models are frequently used in theoretical epidemiology, physiology, and population dynamics to describe the evolution of systems which can be divided into separate compartments, marking the pathways of material flow between compartments and the possible outflow into the inflow from the environment of the system. Generally, the time required for the material flow between compartments cannot be neglected. A model for such system can be visualised as one in which compartments are connected by pipes which material passes through in definite time. Because of the time lags caused by pipes, the model equations for such systems are differential equations with deviating arguments, as opposed to the classical case where model equations are ordinary differential equations. For more details, we refer the reader to $[1,17,21]$.

A concrete example is the radiocardiogram, where the two compartments correspond to the left and right ventricles of the heart, and the pipes between them represent the pulmonary and systematic circulation. Pipes coming out from and returning into the same compartment may represent shunts and the coronary circulation [see 16]. A more simplified equation representing this model is a nonlinear neutral Volterra integrodifferential equation as in [18]. The aim of this paper is to study the controllability problem for such systems.

\footnotetext{
'Department of Mathematics, Bharathiar University, Coimbatore - 641 046, Tamil Nadu, India

(C) Australian Mathematical Society, 1994, Serial-fee code 0334-2700/94
} 
Several authors $[11,14,19,25]$ have studied the theory of functional differential equations. The problem of controllability of linear neutral systems has been investigated in $[7,20]$. Motivation for physical control systems and its importance in other fields can be found in [19, 22]. Angell [2] and Chukwu [9] discussed the functional controllability of nonlinear neutral systems and Underwood and Chukwu [26] studied the null controllability for such systems. Further, Chukwu [10] considered the Euclidean controllability of a neutral system with nonlinear base. Onwuatu [23] discussed the problem for nonlinear systems of neutral functional differential equations with limited controls. Gahl [15] derived a set of sufficient conditions for the controllability of nonlinear neutral systems through the fixed point method developed by Dauer [13].

However Dacka [12] introduced a new fixed-point method of analysis to study the controllability of nonlinear systems with implicit derivative based on the measure of noncompactness of a set and Darbo's theorem. This method has been extended to a larger class of dynamical systems by Balachandran [4, 5]. Anichini et al. [3] studied the problem through the notions of condensing map and measure of noncompactness of a set. They used the fixed-point theorem due to Sadovskii. In this paper, we shall study the controllability of nonlinear neutral Volterra integrodifferential systems with implicit derivative, by suitably adopting the technique of Anichini et al. [3]. The results generalise those results obtained by Balachandran [6] where the nonlinear function $f$ is independent of $\dot{x}$.

\section{Mathematical preliminaries}

We first summarise some facts concerning condensing maps; for definitions and results about the measure of noncompactness and related topics, the reader can refer to the paper of Dacka [12].

Let $X$ be a subset of a Banach space. An operator $T: X \rightarrow X$ is called condensing if, for any bounded subset $E$ in $X$ with $\mu(E) \neq 0$, we have $\mu(T(E))<\mu(E)$, where $\mu(E)$ denotes the measure of noncompactness of the set $E$.

We observe that, as a consequence of the properties of $\mu$, if an operator $T$ is the sum of a compact operator and condensing operator, then $T$ itself is a condensing operator. Further, if the operator $P: X \rightarrow X$ satisfies the condition $|P x-P y| \leq k|x-y|$ for $x, y \in X$, with $0 \leq k<1$, then the operator $P$ is a $\mu$-contractive operator with constant k: that is, $\mu(T(E)) \leq k \mu(E)$ for any bounded set $E$ in $X$. In this case, $P$ has a fixed-point property (Sadovskii [24]). However, the condition $|P x-P y|<|x-y|$ $(x, y \in X)$ is insufficient to ensure that $P$ is a condensing map or that $P$ will admit a fixed point (Browder [8]). The fixed-point property holds in the condensing case (Sadovskii [24]).

Let $C_{n}(I)$ denote the space of continuous $R^{n}$-valued functions on the interval $I$. 
For $x \in C_{n}(I)$ and $h>0$, let

$$
\theta(x, h)=\sup \{|x(t)-x(s)|: t, s \in I \quad \text { with }|t-s| \leq h\},
$$

and write $\theta(E, h)=\sup _{x \in E} \theta \dot{(x, h)}$, so that $\theta(E,$.$) is the modulus of continuity of a$ bounded set $\mathrm{E}$; and let $\Omega$ be the set of functions $\omega: R^{+} \rightarrow R^{+}$that are right continuous and nondecreasing such that $\omega(r)<r$, for $r>0$. Let $I=\left[t_{0}, t_{1}\right]$.

LEMMA 1. [24] Let $X \subset C_{n}(I)$ and let $\beta$ and $\gamma$ be functions defined on $\left[0, t_{1}-t_{o}\right]$ such that $\lim _{s \rightarrow 0} \beta(s)=\lim _{s \rightarrow 0} \gamma(s)=0$. If a mapping $T: X \rightarrow C_{n}(I)$ is given such that it maps bounded sets into bounded sets, and is such that

$$
\theta(T(x), h)<\omega(\theta(x, \beta(h)))+\gamma(h) \quad \text { for all } h \in\left[0, t_{1}-t_{0}\right] \text { and } x \in X
$$

with $\omega \in \Omega$, then $T$ is a condensing mapping.

LEMMA 2. [3, 24] Let $X \subset C_{n}(J)$, let $J=[0,1]$, and let $S \subset X$ be a bounded closed convex set. Let $H: J \times S \rightarrow X$ be a continuous operator such that, for any $\alpha \in J$, the map $H(\alpha, \cdot): S \rightarrow X$ is condensing. If $x \neq H(\alpha, x)$ for any $\alpha \in J$ and any $x \in \partial S$ (the boundary of $S$ ), then $H(1,$.$) has a fuxed point. Finally it is possible to$ show that, for any bounded and equicontinuous set $E$ in $C_{n}^{1}(J)$, the following relation holds:

$$
\mu_{C_{n}^{1}}(E) \equiv \mu_{1}(E) \equiv \mu(D E) \equiv \mu_{C_{n}}(D E)
$$

where $D E=\{\dot{x}: x \in E\}$.

\section{Main result}

Consider the nonlinear neutral Volterra integrodifferential system

$$
\begin{aligned}
\frac{d}{d t}[x(t) & \left.-\int_{0}^{t} C(t-s) x(s) d s-g(t)\right] \\
= & A x(t)+\int_{0}^{t} G(t-s) x(s) d s+B(t) u(t)+f(t, x(t), \dot{x}(t), u(t)),
\end{aligned}
$$

where $x \in R^{n}, u \in R^{m}, C(t)$ is an $n \times n$ continuously differentiable matrix valued function, $G(t)$ is an $n \times n$ continuous matrix, and $B(t)$ is a continuous $n \times m$ matrix valued function, $A$ a constant $n \times n$ matrix and $f$ and $g$ are respectively continuous and continuously differentiable vector functions. Here the control functions are continuous. 
The solution of the system (1) can be written as [27]:

$$
\begin{aligned}
x(t)=Z(t)[x(0) & -g(0)]+g(t)+\int_{0}^{t} \dot{Z}(t-s) g(s) d s \\
& +\int_{0}^{t} Z(t-s)[B(s) u(s)+f(s, x(s), \dot{x}(s), u(s))] d s,
\end{aligned}
$$

where $Z(t)$ is an $n \times n$ continuously differentiable matrix satisfying

$$
\frac{d}{d t}\left[Z(t)-\int_{0}^{t} C(t-s) Z(s) d s\right]=A Z(t)+\int_{0}^{t} G(t-s) Z(s) d s
$$

with $Z(0)=$ identity matrix.

We say that system (1) is controllable if, for every $x(0), x_{1} \in R^{n}$, there exists a control function $\mathrm{u}$ defined on $I=\left[0, t_{1}\right]$ such that the solution of (1) satisfies $x\left(t_{1}\right)=x_{1}$. Define the controllability matrix

$$
W\left(0, t_{1}\right)=\int_{0}^{t_{1}} Z\left(t_{1}-s\right) B(s) B^{*}(s) Z^{*}\left(t_{1}-s\right) d s,
$$

where the star denotes the matrix transpose.

THEOREM. Suppose that the continuity condition on the matrices $G, B, f$ and the continuous differentiability of $C, g$ are satisfied for the system with the following additional conditions:

(i)

$$
\lim _{|x| \rightarrow \infty} \sup \frac{|f(t, x, y, u)|}{|x|}=0
$$

(ii) there exists a continuous nondecreasing function $\omega: R^{+} \rightarrow R^{+}$, with $\omega(r)<r$, such that

$$
|f(t, x, y, u)-f(t, x, z, u)|<\omega(|y-z|) \quad \text { for all }(t, x, y, u) \in I \times R^{2 n} \times R^{m} ;
$$

(iii) the symmetric matrix $W\left(0, t_{1}\right)$ is nonsingular for some $t_{1}>0$.

Then the system (1) is controllable on I.

PROOF. Define the nonlinear transformation

$$
T: C_{m}(I) \times C_{n}^{1}(I) \rightarrow C_{m}(I) \times C_{n}^{1}(I)
$$

by

$$
T(u, x)(t)=\left(T_{1}(u, x)(t), T_{2}(u, x)(t)\right)
$$


where the pair of operators $T_{1}$ and $T_{2}$ is defined by

$$
\begin{array}{r}
T_{1}(u, x)(t)=B^{*}(t) \\
Z^{*}\left(t_{1}-t\right) W^{-1}\left(0, t_{1}\right) \\
\times\left[x_{1}-Z\left(t_{1}\right)(x(0)-g(0))-g\left(t_{1}\right)-\int_{0}^{t_{1}} \dot{Z}\left(t_{1}-s\right) g(s) d s\right. \\
\left.\quad-\int_{0}^{t_{1}} Z\left(t_{1}-s\right) f(s, x(s), \dot{x}(s), u(s)) d s\right], \\
\begin{array}{r}
T_{2}(u, x)(t)=Z(t)(x(0)-g(0))+g\left(t_{1}\right)+\int_{0}^{t} \dot{Z}(t-s) g(s) d s \\
+\int_{0}^{t} Z\left(t_{1}-s\right)\left[B(s) T_{1}(u, x)(s)\right. \\
\left.\quad+f\left(s, x(s), \dot{x}(s), T_{1}(u, x)(s)\right)\right] d s .
\end{array}
\end{array}
$$

Since all the functions involved in the definition of the operator $T$ are continuous, $T$ is continuous. Let

$$
\begin{aligned}
\eta^{0} & =\left(u^{0}, x^{0}\right) \in C_{m}(I) \times C_{n}^{1}(I), \\
\eta & =(u, x) \in\left[C_{m}(I) \times C_{n}^{1}(I)\right] \backslash\{(0,0)\}
\end{aligned}
$$

and consider the equation $\eta^{0}=\eta-\alpha T(\eta)$, where $\alpha \in[0,1]$. This equation can be written equivalently as

$$
\begin{aligned}
& u=u^{0}+\alpha T_{1}(u, x), \\
& x=x^{0}+\alpha T_{2}(u, x) .
\end{aligned}
$$

From condition (i), for any $\varepsilon>0$ there exists $R>0$ such that if $|x|>R$ then $|f(t, x, y, u)|<\varepsilon|x|$. Then (3) gives

$$
|u| \leq\left|u^{0}\right|+k_{1}+a_{1} a_{2}^{2} a_{3} \varepsilon t_{1}|x|
$$

and from (4), by applying Gronwall's inequality, we have

$$
\begin{aligned}
|x| \leq\left[\left|x^{0}\right|+a_{2} \mid x(0)-\right. & g(0) \mid+a_{5}+a_{4} a_{5} t_{1} \\
& \left.+\left(k_{1}+a_{1} a_{2}^{2} a_{3} \varepsilon t_{1}|x|\right) a_{1} a_{2} t_{1}\right] \exp \left(a_{2} \varepsilon t_{1}\right),
\end{aligned}
$$

where

$$
\begin{gathered}
a_{1}=\sup |B(t)|, \quad a_{2}=\sup |Z(t)|, \quad a_{3}=\left|W^{-1}\left(0, t_{1}\right)\right|, \\
a_{4}=\sup |\dot{Z}(t)|, \quad a_{5}=\sup |g(t)|
\end{gathered}
$$

and

$$
k_{1}=a_{1} a_{2} a_{3}\left[\left|x_{1}\right|+a_{2}|x(0)-g(0)|+a_{5}+a_{4} a_{5} t_{1}\right] .
$$


Also put $a_{6}=\sup |\dot{g}(t)|$.

Note that

$$
\begin{aligned}
\frac{d}{d t}\left[T_{2}(u, x)(t)-\int_{0}^{t} C(t-s)\right. & \left.T_{2}(u, x)(s) d s-g(t)\right] \\
=A T_{2}(u, x)(t) & +\int_{0}^{t} G(t-s) T_{2}(u, x)(s) d s \\
& +B(t) T_{1}(u, x)(t)+f\left(t, x(t), \dot{x}(t), T_{1}(u, x)(t)\right) .
\end{aligned}
$$

By applying Gronwall's inequality, we have

$$
\left|T_{2}(u, x)\right| \leq\left[a_{1}\left|T_{1}(u, x)\right| t_{1}+\varepsilon t_{1}|x|+a_{5}\right] \exp \left(A_{0}\right),
$$

where

$$
A_{0}=\int_{0}^{t_{1}}\left|A+C(t-s)+\int_{0}^{t_{1}} G(\eta-s) d \eta\right| d s .
$$

Taking the derivative with respect to $t$, we obtain from (4)

$$
\dot{x}=\frac{d x^{0}}{d t}+\alpha \frac{d}{d t}\left(T_{2}(u, x)(t)\right)
$$

and using Leibnitz's rule that gives

$$
|\dot{x}| \leq\left|\dot{x}^{0}\right|+a\left|T_{2}(u, x)\right|+a_{1}\left|T_{1}(u, x)\right|+\varepsilon|x|+a_{6},
$$

where

$$
a=|A|+\sup |G(t)| \cdot t_{1}+|C(0)|+\sup |\dot{C}(t)| \cdot t_{1} .
$$

Thus from (7), we have

$$
|\dot{x}| \leq\left|\dot{x}^{0}\right|+k_{2}+|x|\left[a_{1}^{2} a_{2}^{2} a_{3} \varepsilon t_{1}\left(a t_{1} \exp \left(A_{0}\right)+1\right)+a \varepsilon t_{1} \exp \left(A_{0}\right)+\varepsilon\right],
$$

where

$$
k_{2}=k_{1}\left[a_{1}\left(a t_{1} \exp \left(A_{0}\right)+1\right)\right]+a a_{5} \exp \left(A_{0}\right)+a_{6} .
$$

From (5), (6) and (8) we have respectively

$$
\begin{aligned}
|u|-a_{1} a_{2}^{2} a_{3} \varepsilon t_{1}|x| & \leq\left|u^{0}\right|+k_{1}, \\
|x|\left[\exp \left(-a_{2} \varepsilon t_{1}\right)-a_{1}^{2} a_{2}^{3} a_{3} \varepsilon t_{1}^{2}\right] & \leq k_{3}+\left|x^{0}\right|
\end{aligned}
$$

where

$$
k_{3}=a_{2}|x(0)-g(0)|+a_{5}+a_{4} a_{5} t_{1}+k_{1} a_{1} a_{2} t_{1}
$$

and

$$
|\dot{x}|-|x|\left[a_{1}^{2} a_{2}^{2} a_{3} \varepsilon t_{1}\left(a t_{1} \exp \left(A_{0}\right)+1\right)+a \varepsilon t_{1} \exp \left(A_{0}\right)+\varepsilon\right] \leq k_{2}+\left|\dot{x}^{0}\right|
$$


Taking the sum of all the above quantities, we obtain

$$
|u|-k|x|+|\dot{x}| \leq\left|u^{0}\right|+\left|x^{0}\right|+\left|\dot{x}^{0}\right|+k_{1}+k_{2}+k_{3},
$$

where

$$
k=a_{1} a_{2}^{2} a_{3} \varepsilon t_{1}\left[1+a_{1} a_{2} t_{1}+a_{1}\left(a t_{1} \exp \left(A_{0}\right)+1\right)\right]+\varepsilon+a \varepsilon t_{1} \exp \left(A_{0}\right)-\exp \left(-a_{2} \varepsilon t_{1}\right) .
$$

Then, for suitable positive constants $p, q$ and $r$, we can write

$$
|u|-[\varepsilon p-\exp (-\varepsilon q)]|x|+|\dot{x}| \leq\left|u^{0}\right|+\left|x^{0}\right|+\left|\dot{x}^{0}\right|+r .
$$

So we divide by $|u|+|x|+|\dot{x}|$ and, from the arbitrariness of $\varepsilon$, we get the existence of a ball $S$ in $C_{m}(I) \times C_{n}^{1}(I)$ sufficiently large such that

$$
|\eta-\alpha T(\eta)|>0 \quad \text { for } \eta=(u, x) \in \partial S .
$$

We want to show that $T$ is a condensing map. To this aim, we note that $T_{1}: C_{m}(I) \rightarrow$ $C_{m}(I)$ is a compact operator and then, if $E$ is a bounded set, $\mu\left(T_{1}(E)\right)=0$. Then it will be enough to show that $T_{2}$ is a condensing operator. For that, let us consider the modulus of continuity of $D T_{2}(u, x)($.$) . Now, for t, s \in I$, we have

$$
\begin{aligned}
\mid D T_{2}(u, x)(t) & -D T_{2}(u, x)(s) \mid \\
\leq \mid & \left|A T_{2}(u, x)(t)-A T_{2}(u, x)(s)\right| \\
& +\left|\int_{0}^{t} G(t-\sigma) T_{2}(u, x)(\sigma) d \sigma-\int_{0}^{s} G(s-\sigma) T_{2}(u, x)(\sigma) d \sigma\right| \\
& +\left|B(t) T_{1}(u, x)(t)-B(s) T_{1}(u, x)(s)\right| \\
& +\left|f\left(t, x(t), \dot{x}(t), T_{1}(u, x)(t)\right)-f\left(s, x(s), \dot{x}(s), T_{1}(u, x)(s)\right)\right| \\
& +\left|C(0) T_{2}(u, x)(t)-C(0) T_{2}(u, x)(s)\right| \\
& +\left|\int_{0}^{t} \dot{C}(t-\sigma) T_{2}(u, x)(\sigma) d \sigma-\int_{0}^{s} \dot{C}(s-\sigma) T_{2}(u, x)(\sigma) d \sigma\right| \\
& +|\dot{g}(t)-\dot{g}(s)| .
\end{aligned}
$$

For the first and last three terms of the right side of (9) we may give the upper estimate as $\beta_{0}(|t-s|)$; and the fourth term by $\omega(|\dot{x}(t)-\dot{x}(s)|)+\beta_{1}(|t-s|)$, with $\lim _{h \rightarrow 0} \beta_{i}(h)=0$. Hence

$$
\theta\left(D T_{2}(u, x), h\right) \leq \omega(\theta(D E, h))+\beta(h),
$$

where $\beta=\beta_{0}+\beta_{1}$. Therefore, by Lemma 1, we get $\theta_{0}\left(D T_{2}(E)\right)<\theta_{0}(D E)$. Hence, from

$$
2 \mu_{1}\left(T_{2}(E)\right)=2 \mu\left(D T_{2}(E)\right)=\theta_{0}\left(D T_{2}(E)\right)<\theta_{0}(D E)=2 \mu(D E)=2 \mu_{1}(E)
$$


it follows that $\mu_{1}\left(T_{2}(E)\right)<\mu_{1}(E)$. Then the existence of the operator $T$ follows from Lemma 2; that is, there exist functions $u \in C_{m}(I)$ and $x \in C_{n}^{1}(I)$ such that $T(u, x)=(u, x)$ that is

$$
u(t)=T_{1}(u, x)(t), \quad x(t)=T_{2}(u, x)(t) .
$$

These functions are the required solutions. Further, it is easy to verify that the function $x(\cdot)$ given above of the system (1) satisfies $x\left(t_{1}\right)=x_{1}$ for every $x(0) \in R^{n}$. Hence the system ( 1$)$ is controllable.

REMARK 1. If we assume that the nonlinear function in (1) also satisfies the Lipschitz condition with respect to the state variable, then we can obtain the unique response determined by any control.

REMARK 2. The result of Theorem 1 still holds if we replace (i) by

$$
|f(t, x, \dot{x}, u)| \leq \alpha(t)|x|+\beta(t),
$$

where $\alpha$ and $\beta$ are continuous functions.

EXAMPLE. In the nonlinear neutral Volterra integrodifferential system (1), take

$$
\begin{gathered}
C(t-s)=e^{-(t-s)}, \quad g(t)=e^{-t}, \quad A=1, \quad G(t-s)=-e^{-(t-s)}, \\
B(t)=e^{-2 t} \quad \text { and } \quad f=\frac{\log x}{\sqrt{1+u^{2}}}+\operatorname{arctg} \dot{x} .
\end{gathered}
$$

It is easy to see that $Z(t)=2 e^{t}-1$ satisfies (2) and

$$
\begin{aligned}
W\left(0, t_{1}\right) & =\int_{0}^{t_{1}} Z\left(t_{1}-s\right) B(s) B^{*}(s) Z^{*}\left(t_{1}-s\right) d s \\
& =\int_{0}^{t_{1}} e^{-4 s}\left[4 e^{2\left(t_{1}-s\right)}-4 e^{\left(t_{1}-s\right)}+1\right] d s \\
& =\frac{2}{3} e^{2 t_{1}}-\frac{4}{5} e^{t_{1}}+\frac{1}{4}-\frac{7}{60} e^{-4 t_{1}}>0 \quad \text { for some } t_{1}>0 .
\end{aligned}
$$

Furthermore

$$
\begin{aligned}
|f(t, x, y, u)-f(t, x, z, u)| & =|\operatorname{arctg} y-\operatorname{arctg} z| \\
& <\operatorname{arctg}|y-z| \quad \text { if } y \neq z
\end{aligned}
$$

and

$$
\lim _{|x| \rightarrow \infty} \frac{|f(t, x, y, u)|}{|x|}=0,
$$

so the hypotheses of Theorem 1 are satisfied. Hence the system is controllable. 


\section{Acknowledgement}

This research was supported by CSIR, New Delhi.

\section{References}

[1] D. H. Anderson, Compartmental modeling and tracer kinetics (Springer-Verlag, New York, 1983).

[2] T. S. Angell, "On controllability for nonlinear hereditary systems; a fixed point approach", Nonlinear Analysis, Theory, Methods and Appl. 4 (1980) 529-545.

[3] G. Anichini, G. Conti and P. Zecca, "A note on controllability of certain nonlinear systems", Note di Matematica 6 (1986) 99-111.

[4] K. Balachandran, "Global and local controllability of nonlinear systems", IEE Proc. Pt D 132 (1985) 13-17.

[5] K. Balachandran, "Controllability of perturbed nonlinear systems", IMA J. Math. Control and Infor. 6 (1989) 253-258.

[6] K. Balachandran, "Controllability of neutral Volterra integrodifferential systems", J.Austral. Math. Soc., Ser. B 34 (1992) 18-25.

[7] H. T. Banks and G. A. Kent, "Control of functional differential equations of retarded and neutral type to target sets in function space", SIAM J. Control 10 (1972) 567-593.

[8] F. E. Browder, "Nonexpansive nonlinear operators in Banach spaces", Proc. Nat. Acad. Sci 54 (1965) 1041-1044.

[9] E. N. Chukwu, "Functional inclusion and controllability of nonlinear neutral functional differential systems", J. Optim. Theory Appl. 29 (1979) 291-300.

[10] E. N. Chukwu, "On the Euclidean controllability of a neutral system with nonlinear base", Nonlinear Analysis, Theory, Methods and Appl. 11 (1987) 115-123.

[11] E. N. Chukwu and H. C. Simpson, "Perturbations of nonlinear systems of neutral type", J. Diff. Eqns 82 (1989) 28-59.

[12] C. Dacka, "On the controllability of a class of nonlinear systems", IEEE Trans. Autom. Control AC-25 (1980) 263-266.

[13] J. P. Dauer, "Nonlinear perturbations of quasilinear control systems", J. Math. Anal. Appl. 54 (1976) 717-725.

[14] E. Fuchs, "The degeneracy property in linear autonomous functional differential equations of neutral type", J. Math. Anal. Appl. 90 (1983) 527-549.

[15] R. D. Gahl, "Controllability of nonlinear systems of neutral type”, J. Math. Anal. Appl. 63 (1978) 33-42.

[16] I. Györi, "Delay differential and integrodifferential equations in biological compartmental models", Systems Sci., (Wroclaw) Poland 8 (1982) 167-187.

[17] I. Györi, "Connections between compartmental systems with pipes and integrodifferential equations", Math. Model 7 (1986) 1215-1238.

[18] I. Györi and J. Wu, "A neutral equation arising from compartmental systems with pipes", $J$. Dynamics and Diff. Eqns 3 (1991) 289-311.

[19] J. K. Hale, Theory of functional differential equations (Springer-Verlag, New York, 1977).

[20] M. Q. Jacobs and C. E. Langenhop, "Criteria for function space controllability of linear neutral systems", SIAM J. Control and Optim. 14 (1976) 1009-1048.

[21] J. A. Jacquez, Compartmental analysis in biology and medicine (Springer-Verlag, New York, 1972). 
[22] D. A. O'Connor, "State controllability and observability for linear neutral systems", Dr. Sc. Thesis, Washington University, 1978.

[23] J. U. Onwuatu, "On the null controllability in function space of nonlinear systems of neutral functional differential equations with limited controls", J. Optim. Theory Appl. 42 (1984) $327-$ 420.

[24] B. J. Sadovskii, "Limit compact and condensing operators", Russian Math. Surveys 27 (1972) 85-155.

[25] G. Tadmore, "Functional differential equations of retarded and neutral type; analytic solutions and piecewise continuous controls", J. Diff. Eqns 51 (1984) 151-181.

[26] R. G. Underwood and E. N. Chukwu, "Null controllability of nonlinear neutral differential equations", J. Math. Anal. Appl. 129 (1988) 326-345.

[27] J. Wu, "Globally stable periodic solutions of linear neutral Volterra integrodifferential equations", J. Math. Anal. Appl 130 (1988) 474-483. 